\title{
A Geometry of Networks
}

\author{
Yechiam Yemini \\ Department of Computer Science \\ Columbia University \\ 450 Computer Science Building \\ 1214 Amsterdam Avenue, Mailcode: 0401 \\ New York, New York 10027-7003 \\ yemini@cs. columbia.edu
}

\begin{abstract}
This presentation will describe a coordinate geometry of networks and its applications to mobility, security, overlays and traffic management. Given a network graph, one can construct an abstract group and associate elements of the group with graph nodes to provide "coordinates". Much like Cartesian coordinates, a route may be simply computed from the coordinates of the source and destination. Given a metric of link "length" (e.g., delay, utilization), one may easily select shortest-distance routes. Furthermore, this route selection may vary for each source-destination stream, or even for each packet. For example, unlike current networks, one may pursue dispersion-routing where a stream of packets is routed over multiple paths to load-balance traffic. The coordinates geometry admits dynamic topology changes and may thus be used to support various forms of mobility, including mobile adhoc networks, or dynamic deployment of virtual-machines through cloud infrastructures. A given network may admit multiple independent geometry overlays. Each such overlay can serve as a VPN to protect access to respective nodes. Finally, geometry overlays may be flexibly layered over each other, permitting simple scalability, applications-specific-networks and flexible private networks.
\end{abstract}

